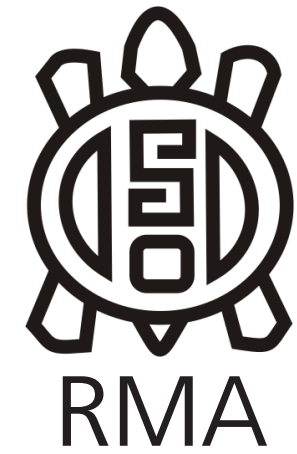

Dossier

\title{
Discutiendo unidades de análisis para el estudio de artefactos líticos
}

\author{
Discussion on units of analysis for lithic artifacts studies
}

Federico Restifo*

*CONICET-Instituto de Arqueología, Universidad de Buenos Aires, Argentina. E-mail: federicorestifo@gmail.com

\begin{abstract}
Resumen
Se discuten criterios para la elección o construcción de unidades de análisis en estudios líticos. Asumiendo que los conjuntos líticos representan un continuo de variación morfológica y métrica, su ordenamiento a partir de diferentes unidades es responsabilidad del investigador dado que no tienen una segmentación inherente. Se resalta el criterio de elección o construcción de unidades según su relevancia para responder a una pregunta de investigación. Se argumenta que la confiabilidad y validez de las unidades de análisis es crucial para el proceso de investigación.
\end{abstract}

Palabras clave: Unidades de análisis; Variación; Artefactos líticos; Clasificación.

\begin{abstract}
Criteria for the choice or construction of units of analysis in lithic studies are discussed. Assuming that the lithic assemblages represent a continuum of morphological and metric variation, since they do not have an inherent segmentation, their ordering from different units is the responsibility of the researcher. The main criterion of choice or construction of units is their relevance regarding a research question. It is argued that the reliability and validity of the units of analysis is crucial for the research process.
\end{abstract}

Keywords: Units of analysis; Variation; Lithic artifacts; Classification.

\section{Introducción}

Considerando el aspecto teórico-metodológico de los estudios líticos en Argentina, la elección o construcción de unidades de análisis para el estudio de la variación en artefactos no ha sido motivo de discusiones extendidas, más allá de algunas contribuciones (Aschero 1975, Orquera y Piana 1992, Borrero 1993, Hocsman 2009, Cardillo 2009). En este sentido, en el plano internacional, particularmente en Estados Unidos, se destaca un debate de larga data acerca de la naturaleza empírica o abstracta de las unidades empleadas para la clasificación de conjuntos de artefactos, particularmente los "tipos" (Spaulding 1953, Ford 1954). Dicho debate fue retomado desde los años '70 en el marco de la arqueología evolutiva, postulando que los tipos son unidades definidas por un observador, y como tales, su elección debería basarse en su potencial o relevancia para responder a un interés o pregunta de investigación particular (Ford 1954, Dunnell 1977, O'Brien y Lyman 2002). De no resultar aptos para abordar un tema de investigación específico, los tipos no serían unidades necesarias. Esto también se ha planteado recientemente en el marco de la arqueología australiana, discutiendo las limitaciones de un abordaje tipológico para captar la variación morfológica de los artefactos a lo largo de su vida útil (Hiscock 2007).
Este criterio de relevancia también puede pensarse para el caso de unidades de menor nivel o inclusividad, como los atributos. Su elección también depende del potencial para abordar un interrogante de investigación en particular (O’Brien y Lyman 2002). Por ejemplo, el análisis de la inversión de trabajo o el de sistemas de armas demandará la selección (o construcción) y análisis de diferentes conjuntos de atributos, lo que también implicará dejar otros atributos de lado.

En síntesis, a partir de estos planteos sobre unidades -artefactos y atributos específicamente- surge la pregunta principal de este trabajo: ¿bajo qué criterios elegimos o construimos las unidades que empleamos en nuestros análisis de artefactos líticos? Tomando en cuenta el contexto del Primer Congreso Argentino de Estudios Líticos en Argentina, considero que se trata de una buena oportunidad para tal discusión.

\section{Acerca del abordaje de la variación}

El punto fundamental, y en el que acordamos con diferentes colegas, más allá de analizar los artefactos líticos a la luz de diferentes lineamientos teóricos, es el hecho de que la variación que se observa en los conjuntos líticos se presenta a modo de un continuo 
(Aschero 1975, Hocsman 2009, Cardillo 2009, Restifo 2013 a, Heider y Rivero 2018). De este modo, se asume que en un conjunto lítico ningún artefacto es idéntico a otro, por lo que cualquier segmentación o agrupamiento de piezas bajo criterios de similitud emerge como una construcción de quien analiza (Dunnell 1977, O’Brien y Lyman 2002, Shott 2003). Este planteo constituye una lectura de la tecnología lítica desde la óptica de la ontología materialista (Sober 1992). A su vez, esto se diferencia de otra ontología, denominada esencialismo.

Brevemente, desde el esencialismo se asume que la realidad que se analiza se encuentra naturalmente segmentada en grupos discretos, integrados por especímenes que comparten un conjunto de rasgos entre sí, o bien una esencia, que los hace diferentes de los especímenes que integran otros grupos (Mayr 2006). Ante esta realidad, la labor del investigador sólo se reduce al descubrimiento de dichos grupos (Spaulding 1953).

Sin embargo, desde el materialismo, al considerarse a la realidad como un continuo de variación, integrada por especímenes diferentes entre sí, y al no existir ninguna segmentación inherente en grupos, es responsabilidad del analista la construcción y/o elección crítica de unidades de análisis para la segmentación de un conjunto. Derivado de lo expuesto, en las próximas secciones discuto algunos criterios para la elección o construcción de unidades de análisis en estudios líticos.

\section{¿Qué es una unidad de análisis?: jerarquía y clases de unidades}

Siguiendo a Ramenofsky y Steffen (1998) las unidades de análisis pueden definirse como medios a partir de los cuales se especifica un rango de variación que es relevante para determinados intereses de investigación. A su vez, adhiriendo a un sentido amplio de esta definición, el rango de variación que describen las unidades de análisis puede ampliarse o restringirse de acuerdo con la escala de observación. Por lo tanto, las unidades de análisis pueden organizarse dentro de un esquema jerárquico o taxonómico, en el que la inclusividad decrece a medida que se desciende en la jerarquía. De este modo, conjunto, artefacto y atributo representan diferentes unidades de análisis, especificando diferentes rangos de variación o recortes, y entre las cuales el atributo es la unidad de menor inclusividad, respecto del artefacto (Muscio y López 2007:118), y más aún respecto del conjunto. En consecuencia, construir o elegir unidades de análisis es, en última instancia, realizar recortes en el horizonte de variación continua.

Asimismo, es clave distinguir entre unidades empíricas y unidades ideacionales (Dunnell 1977, O'Brien y Lyman 2002). Las unidades empíricas son aquellas físicamente perceptibles, tales como el artefacto lítico en nuestro caso. Por su parte, las unidades ideacionales son aquellas creadas para efectuar mediciones sobre las primeras. Entre las unidades ideacionales comúnmente se mencionan a las unidades de milímetros, grados o gramos ( $\mathrm{O}$ 'Brien y Lyman 2002). A modo de ejemplo, pueden mencionarse también los intervalos de tamaños, tales como "muy pequeño", "pequeño", "mediano-pequeño", etc. (Aschero 1975), con la particularidad de que miden la variación de una dimensión continua mediante categorías discretas.

Asimismo, los atributos, y sus diferentes variantes o estados, presentados en el Ensayo para una clasificación morfológica de artefactos líticos, propuesta de uso corriente en Argentina (Aschero 1975), también pueden considerarse unidades ideacionales. Esto se explica, principalmente, por tratarse de construcciones. Es decir, categorías diseñadas por un investigador. Asimismo, en tanto construcciones tienen un propósito, el cual consiste en medir la variación de un rasgo particular de un artefacto. Como ejemplo, podemos considerar la variación en la morfología de la base de una punta de proyectil, la cual puede reflejarse según categorías como "recta", "escotada" o "acuminada", por nombrar sólo algunas variantes. Las frecuencias de una u otra de las categorías de la morfología de la base, observadas en un conjunto, darán lugar a una medida de la variación en dicho rasgo. Sin embargo, si esas categorías no son suficientes para dar cuenta de la variabilidad en artefactos particulares de un conjunto, entonces será necesario buscar nuevas categorías, o bien crearlas. Otro camino posible sería el de abordar el estudio de la morfología del artefacto, cualesquiera que sean los atributos de interés, considerando su naturaleza continua, a partir de análisis morfométricos (Cardillo 2009, Heider y Rivero 2018).

Siguiendo con el ejemplo, respecto del atributo morfología de la base, y considerando sus respectivos estados posibles, puede hablarse de "variable". En este caso, la variable "morfología de la base". Si entendemos como variable a todo aquello que es susceptible de adquirir distintos estados, entonces no sólo los atributos constituyen variables, sino también los artefactos, por contemplar diferentes posibilidades de clasificación, en este caso a partir de unidades ideacionales, es decir construcciones de un investigador. Como ejemplo pueden mencionarse los tipos de artefactos definidos por atributos específicos (e.g. raederas, cuchillo).

\section{¿En base a qué criterio se eligen o construyen unidades de análisis?}

Asumiendo que los conjuntos líticos se presentan a modo de un continuo de variación, no hay otra guía para la elección o construcción de unidades de análisis más que la pregunta o interés de investigación que estamos abordando (Nielsen 1995, Ramenofsky y Steffen 1998, O'Brien y Lyman 2002). A partir de lo dicho se desprende que las unidades que empleamos para clasificar nuestros 
conjuntos pueden variar según el interrogante, y en consecuencia pueden existir tantas clasificaciones como problemáticas abordadas (ver Nielsen 1995). Dicho en otras palabras, al no existir un orden inherente al fenómeno bajo estudio, tampoco existe una clasificación única y definitiva. Esto queda claro al pensar diferentes temáticas de análisis lítico macroscópico.

Por ejemplo, la evaluación de la inversión de trabajo tomará en cuenta un recuadro de la variación total de un conjunto lítico, diferente del que puede considerar el análisis de sistemas de armas. Si bien en ambos casos se consideran unidades empíricas, artefactos líticos particularmente, en el primer caso el conjunto puede clasificarse en artefactos de filo retocado (clasificados como raspador, raedera, cuchillo), bifaces, así como puntas de proyectil, entre otras clases. A su vez, se tomarán en cuenta atributos -unidades ideacionales de menor nivel- como la forma base, situación y extensión de los lascados sobre las caras, presencia/ausencia de adelgazamiento bifacial, etc. (Hocsman y Escola 20062007). En el segundo caso, el análisis se va a acotar a los especímenes clasificados macroscópicamente como puntas de proyectil, o bien "cabezales líticos" (Ratto 2003), y se tomarán en cuenta atributos métricos y cualitativos -unidades ideacionales también- vinculados con la performance de los artefactos como proyectiles (Ratto 2003). Al margen de las diferencias de unidades ideacionales, en ambos casos se emplea un criterio de "validez", seleccionando unidades por su relevancia para abordar una problemática de trabajo (Ramenofsky y Steffen 1998).

Lo que se deduce de estos breves ejemplos es que cada pregunta de investigación, ya sea sobre inversión de trabajo o sistemas de armas, toma en cuenta un recuadro particular de la variación total de un conjunto lítico. Respecto de la variación restante del conjunto, no considerada a la luz de las problemáticas planteadas, debe tenerse en cuenta que puede recobrar valor en el marco de otros interrogantes de trabajo (Dunnell 1977).

Asimismo, la elección de las unidades de análisis debe someterse a prueba según su carácter operativo. Con esto me refiero a la "confiabilidad" de las unidades (Ramenofsky y Steffen 1998), es decir, la consistencia con la cual es definida una unidad. Si su definición es lo suficientemente específica y clara, la unidad dará lugar a medidas precisas. Por lo tanto, su aplicación va a generar resultados comparables. En este sentido, el conjunto de atributos con sus diferentes variantes, presentados en la propuesta de Aschero (1975), resulta confiable para la descripción de piezas líticas, favoreciendo la comparación entre conjuntos, cualquiera sea la escala espacial de su distribución. Por ejemplo, para el caso de la inversión de trabajo, las diferentes categorías para evaluar la situación y extensión del retoque sobre las caras, así como la variación de formas base, entre otras, pueden resultar válidas, así como confiables.

Por su parte, en el nivel del artefacto, una unidad resultaría confiable si su definición es lo suficientemente específica y clara para discriminar un artefacto, o grupo de artefactos, de otro. A modo de ejemplo, un tipo de punta de proyectil resulta confiable cuando se construye a partir de un conjunto de atributos o estados particulares de los mismos, que lo diferencian de otro tipo. El mismo criterio aplica para la segmentación de otros grupos de artefactos, como aquellos de filo retocado. Si la definición de estas unidades carece de precisión y claridad, entonces la posibilidad de errores de medición aumenta, derivando, por ejemplo, en errores de clasificación entre diferentes observadores. Desde luego, una unidad que no reúne tales condiciones de precisión y claridad pierde su validez y confiabilidad.

\section{Breve alusión a un caso de estudio en el Noroeste Argentino}

A modo de ejemplo, en mi investigación de tesis de doctorado (Restifo 2013 b) analicé, entre otros aspectos, la inversión de trabajo en conjuntos líticos de la puna de la provincia de Salta, particularmente del valle de San Antonio de los Cobres y de la cuenca de Pastos Grandes, correspondientes al Holoceno temprano y medio (ca. 10000-4000 AP). La intención de dicho análisis tuvo sentido en el marco de una hipótesis derivada de un marco teórico ecológico evolutivo (Bousman 1993). De este modo, se planteó como expectativa que la inversión de trabajo en tecnología lítica aumentaría en contextos de mayores presiones selectivas para la adaptación humana, reflejando estrategias tecnológicas que involucren mayores costos en la producción de artefactos.

Siguiendo el ejemplo, me centraré en lo trabajado en relación a artefactos formatizados. A los fines analíticos seleccioné un conjunto de atributos codificados en la propuesta de clasificación de Aschero (1975), los que constituyeron unidades ideacionales, por resultar adecuadas para medir la inversión de trabajo en la secuencia temporal Holoceno temprano/medio, a saber: forma base, situación de los lascados sobres las caras y extensión de los lascados sobre las caras. A estos atributos agregué la clase de materia prima, el conteo de la cantidad de filos por artefacto formatizado, y la clase técnica (Hocsman 2009). Cada uno de los atributos posee sus diferentes estados o variantes, los cuales permiten hacer efectiva la clasificación.

Las tendencias observadas, a partir de la consideración de los diferentes atributos, permitieron discutir la hipótesis propuesta. El atributo "forma base" fue clave, destacándose un cambio tecnológico fundamental como es la proliferación de la tecnología de hojas en la puna de Salta, hacia finales del Holoceno medio, lo que fue vinculado con un aumento en la inversión de 
trabajo (Restifo 2013 b). En este caso, si bien desde otra terminología, lo que evalué fue el "requerimiento de extracción de forma base" (Hocsman y Escola 2006-2007), el cual constituye otra unidad ideacional para medir la inversión de trabajo en tecnología lítica.

Ahora bien, otra cuestión relevante es ¿Sobre qué otras unidades se deberían medir estos atributos? Dado que un conjunto de artefactos líticos formatizados no constituye una totalidad homogénea en términos morfológicos, resulta interesante indagar en la inversión de trabajo según clases de artefactos. Entonces, ¿en base a qué criterios se seleccionan o crean esas clases de artefactos?

Una primera opción es la distinción de grupos tipológicos tal como se planteó en la propuesta clasificatoria de uso corriente en nuestro país (Aschero 1975, 1983), y que constituye un lenguaje común por lo menos en la comunidad arqueológica argentina. Sin embargo, dado que el abordaje de la inversión de trabajo no necesariamente justifica la distinción de clases de artefactos a nivel específico, me incliné por el uso de otras unidades, en este caso de mayor inclusividad (ver Cardillo 2009). De este modo, siguiendo el principio de "clasificación materialista" (Hiscock 2007), utilicé clases de artefactos definidas a partir de atributos puramente observables a nivel macroscópico. Así, dejando de lado el componente funcional hipotético de los grupos tipológicos, utilicé la categoría "artefacto de filo retocado", la cual podría incluir en su dominio empírico a diferentes piezas que hayan cumplido funciones de corte, raspado o raído, entre otras.

Otras de las categorías utilizadas fueron "biface", "cabezal lítico" (Ratto 2003) y "artefacto de formatización sumaria" (Aschero 1975), esta última por remitirse específicamente a atributos observables macroscópicamente. Si bien algunas categorías pueden resultar altamente inclusivas, fueron adecuadas para arribar a patrones generales de variación temporal en la inversión de trabajo en los conjuntos líticos analizados.

\section{A modo de cierre}

El proceso de construcción o elección de unidades de análisis resulta fundamental en una investigación, dado que es el que la encamina, estableciendo las pautas para la generación de datos adecuados para discutir una hipótesis, o bien una pregunta de investigación. Es decir, dentro de las etapas del método hipotético deductivo, constituye un paso anterior a la generación de datos. En este marco, es importante distinguir entre lo que es la definición de unidades de análisis por "extensión" y por "intención" (Dunnell 1977, O’Brien y Lyman 2002). La definición por extensión es aquella que pone énfasis en la construcción de unidades a partir del criterio de atributos compartidos según lo que puede observarse en un conjunto con determinado número de especímenes.
El método tipológico clásico utiliza este criterio de definición, ya que construye unidades -tipos- en base a los atributos de mayor recurrencia en un conjunto dado.

A diferencia de las unidades definidas por extensión, las unidades definidas por intención dependen de su relevancia para resolver una problemática de investigación y derivan de la teoría, o bien de un modelo particular acerca de cómo ocurre cierto fenómeno. Su construcción o elección está guiada plenamente por la pregunta de investigación. Por ejemplo, los atributos mencionados líneas arriba para la asignación funcional de cabezales líticos constituyen unidades definidas por intención, en tanto surgen en función de interrogantes específicos. Asimismo, están sustentados por un modelo que predice los atributos que debe reunir tal o cual sistema de armas, en función de información etnográfica y experimentos, entre otros aspectos (ver Ratto 2003). Del mismo modo, ciertas unidades empleadas para diferenciar clases de artefactos, como "artefacto de filo retocado", discutida en la sección anterior, también son consecuencia de una definición por intención, dado que la extensión del rango de variación que contemplan (sea más o menos inclusivo) responde a un interés particular de trabajo, favoreciendo la identificación de tendencias de cambio en una secuencia temporal de largo plazo.

Por su parte, la selección de los atributos para evaluar la inversión de trabajo en la manufactura de artefactos líticos tiene también un sustento teórico en tanto se comprueba su relación directa con dicho comportamiento. Por ejemplo, la clase de forma base adquiere pertinencia al determinarse los mayores costos involucrados en la extracción sistemática de hojas, en relación con la obtención de lascas sin un patrón morfológico distintivo. Esto se sustenta a partir de la secuencia de reducción más compleja para el caso de las hojas, involucrando una mayor cantidad de etapas, tales como la elección de una unidad de materia prima determinada, la generación de una arista, la preparación de plataformas, entre otras (Bellelli y Nami 1993). La base actualística experimental que permitió arribar a estas consideraciones constituye la teoría (en este caso de rango medio) a partir de la cual el análisis de la forma base adquiere relevancia -validez- en un análisis de inversión de trabajo.

Ambos modos de definición de unidades, por extensión y por intención, sin embargo, no son mutuamente excluyentes, sino que pueden resultar pasos complementarios en un proceso de investigación. En un análisis de sistemas de armas, por ejemplo, la definición de tipos de puntas de proyectil, de acuerdo a sus atributos compartidos, puede constituir un primer paso para ordenar un conjunto. Pero esto es independiente de la función de las puntas de proyectil en relación con sistemas de armas, la cual se evalúa a partir de unidades ideacionales como la "superficie de refuerzo", "aerodinámica", "penetración", entre otras (Ratto 2003), pudiendo darse el caso de una 
misma asignación funcional para diferentes tipos (Restifo 2013 a). Asimismo, otro camino podría ser el de trabajar en el nivel del atributo, y evaluar cómo se agrupan las puntas de proyectil en el espacio multivariado según las variables diagnósticas de sistemas de armas, a partir de un análisis de componentes principales (Ratto 2003: 216).

Desde luego, esto no invalida el procedimiento tipológico, el cual resulta adecuado para establecer un orden. Sin embargo, es relevante considerar que este último, al menos desde las propuestas de Argentina, ha sido justificado bajo la necesidad de ordenar un continuo morfológico "de acuerdo a las características que el propio material ofrece" (Aschero 1975: 48, Hocsman 2009). Si se asume que la elección o construcción de unidades de análisis es parte de un proceso particular de cada problemática de estudio, entonces el criterio de ordenamiento estaría dado por el interrogante de investigación más que por las características del propio material. Si la muestra disponible es la adecuada para abordar la problemática de estudio específica es otra cuestión, que a su vez admite solución.

En consecuencia, nuestra pregunta de investigación será un principio determinante para seleccionar un conjunto de atributos, que luego puedan conformar unidades de mayor nivel de inclusividad, independientemente de sí se trata o no de los más recurrentes. El criterio central está dado por la validez y la confiabilidad. Por último, si las unidades de análisis disponibles no satisfacen dichos criterios, respecto de un interrogante de trabajo en particular, será necesario buscar, o bien crear, nuevas unidades. Tal proceso de creación es parte fundamental del trabajo de investigación científica.

Ciudad Autónoma de Buenos Aires, 14 de Abril de 2019

\section{Agradecimientos}

Agradezco al CONICET por el apoyo a mi investigación en el Noroeste Argentino. A las y los colegas asistentes al simposio "Avances y desafíos en torno a los abordajes cuali-cuantitativos aplicados a los estudios líticos", llevado a cabo en el Primer Congreso Argentino Estudios Líticos en Arqueología, por permitir el mejor ambiente para la discusión de ideas. Al Dr. Marcelo Cardillo, con quien coordinamos dicho simposio. A los Dres. Juan P. Carbonelli, Rodolphe Hoguin y Luis Borrero por la lectura crítica del manuscrito de este trabajo. A quienes evaluaron el trabajo, por sus comentarios enriquecedores.

\section{Bibliografía}

Aschero, C. (1975). Ensayo para una clasificación morfológica de los instrumentos líticos aplicada a estudios tipológico comparativos. Informe al CONICET. Manuscrito inédito.
Aschero, C. (1983). Ensayo para una clasificación morfológica de artefactos líticos. Apéndice A y B. Informe presentado al CONICET. Manuscrito inédito.

Bellelli, C. y H. Nami (1994). Hojas, experimentos y análisis de desechos de talla. Implicaciones arqueológicas para la Patagonia Centro-Septentrional. Cuadernos del INAPL 15:199-224.

Borrero, L.A. (1993). Artefactos y evolución. Palimpsesto 3: $15-32$.

Bousman, B. (1993). Hunter gatherer adaptations, economic risk and tool design. Lithic Technology 18: 59- 86.

Cardillo, M. (2009). Perspectivas Darwinianas para el análisis de la tecnología lítica en argentina. En M.C. Barboza (Ed.) 150 años después... La vigencia de la teoría evolucionista de Charles Darwin (pp. 17-32). Rosario: Universidad Nacional de Rosario.

Dunnell, R. (1977). Prehistoria moderna. Introducción sistemática al estudio de la arqueología prehistórica. Madrid: Ediciones Istmo.

Ford, J. (1954). On the concept of types. American Anthropologist 56 (1): 42-57.

Heider, G. y D. Rivero. (2018). Estudios morfométricos aplicados a puntas de proyectil lanceoladas del Holoceno temprano-medio en sierras y llanuras pampeanas de Argentina. Latin American Antiquity, 29(3): 572-590.

Hiscock, P. (2007). Looking the other way: a materialist/ technological approach to classifying tools and implements, cores and retouched flakes. En S. McPherron (Ed.) Tools versus cores? alternative approaches to stone tool analysis, (pp. 198-222). New Castle: Cambridge Scholar Publishing.

Hocsman, S. (2009). Una propuesta de aproximación teórico-metodológica a conjuntos de artefactos líticos tallados. En Barberena, R., Borrazzo, K. y L. Borrero (Eds.) Perspectivas Actuales en Arqueología Argentina (pp. 271-302). Buenos Aires: CONICET.

Hocsman, S. y P. Escola. (2006-2007). Inversión de trabajo y diseño en contextos líticos agro-pastoriles (Antofagasta de la Sierra, Catamarca). Cuadernos del INAPL 21: 75-90.

Mayr, E. (2006). Typological versus population thinking. En E. Sober (Ed.) Conceptual issues in evolutionary biology. (pp. 325-328). Londres (Inglaterra): The MIT Press.

Muscio, H. y G. López. (2007). Unidades de análisis arqueológicas en el estudio evolutivo de adaptaciones con 
economías de producción de alimentos: Un exámen de las arqueofaunas de la quebrada de Matancillas. Shinkal 7: 111-134.

Nielsen, A. (1995). El pensamiento Tipológico como Obstáculo para la Arqueología de los Procesos de Evolución en Sociedades sin Estado. Comechingonia 8: 21-45.

O'Brien, M. y L. Lyman. (2002). The epistemological nature of archaeological units. Anthropological Theory 2 (1): 37-56.

Orquera, L. A. y E. Piana. (1992). Normas para la descripción de objetos arqueológicos de piedra tallada. Centro Austral de Investigaciones Científicas.

Ramenofsky, A. F., \& Steffen, A. (1998). Units as tools of measurement. En Ramenofsky, A. y A. Steffen (Eds.) Unit issues in Archaeology, (pp. 3-17). University of Utah Press.

Ratto, N. (2003). Estrategias de caza y propiedades del registro arqueológico en la Puna de Chaschuil (Departamento de Tinogasta, Catamarca, Argentina). Tesis doctoral. Facultad de Filosofía y Letras. Universidad de Buenos Aires, Argentina.
Restifo, F. (2013) a. Herramientas teórico-metodológicas para el análisis del cambio en la tecnología lítica: aportes desde una perspectiva evolutiva. Arqueología 19: 125149.

Restifo, F. (2013) b. Tecnología lítica en la puna de la provincia de Salta a lo largo del Holoceno temprano y medio: Patrones de variación y procesos de cambio. Tesis doctoral. Facultad de Filosofía y Letras, Universidad de Buenos Aires, Argentina.

Shott, M. (2003). Time as Sequence, Type as Ideal: Whole-Object Measurement of Biface Size and Form in Midwestern North America. En: Soressi, M. y H. Dibble (Eds.) Multiple Approaches to the Study of Bifacial Technologies (pp. 251-271). Pennsylvania: University of Philadelphia.

Sober, E. (1992). Evolution, population thinking and essentialism. En M. Ereshefzky (Ed.) The units of evolution. Essays on the nature of species (pp. 247-278). Cambridge: The MIT Press.

Spaulding, A. (1953). Statistical techniques for the discovery of artifact types. American Antiquity 18 (4): 305-313. 